\title{
Environmental impacts of alternative cement binders
}

Sabbie A. Miller ${ }^{a}{ }^{\dagger}$, Rupert J. Myers ${ }^{b, c}$

${ }^{a}$ Department of Civil and Environmental Engineering, University of California, Davis

2001 Ghausi Hall, One Shields Ave, Davis, CA, 95616

${ }^{\mathrm{b}}$ School of Engineering, University of Edinburgh

Sanderson Building, Robert Stevenson Road, King's Buildings, Edinburgh, EH9 3FB

${ }^{\mathrm{c}}$ Current address: Department of Civil and Environmental Engineering, Imperial College London

Skempton Building, South Kensington Campus, London, SW7 2AZ

$\dagger$ Corresponding Author: T +1 530754 6407, E sabmil@ucdavis.edu

\section{This PDF file includes:}

Supplementary Methods, pages S2 - S5

Supplementary Data Tables S1 - S15

Supplementary Figures S1 - S10

Supplementary References, pages S5 - S6 


\section{S.1. Methodological Summary}

In this work, environmental impact assessments of various cement binders were conducted. To perform these assessments, the heat requirements and raw material inputs necessary to form reactive cement phases were quantified. These inputs were combined with data on conventional production methods for portland cement (PC) to assess and compare the production of the various cement binders examined. We used thermodynamics as a means to model inventory data for cement binder production because many of the systems analyzed in this work are not being produced at an industrial scale. This approach facilitates rational and consistent comparisons of both novel and commercially used cement systems. We used the United States Environmental Protection Agency's TRACI scheme to quantify cradle-to-gate production environmental impacts of these binders. Reactions between the various cements examined here and either water or $\mathrm{CO}_{2}$, i.e., curing, to form $1 \mathrm{~kg}$ of each binder, were assessed. These binders were assessed here separately from many admixtures that are typically utilized in cement-based materials, such as superplasticizers, due to the high variety of admixtures in common use. Table S1 summarizes cement chemistry notation used.

The stages of analysis conducted in this work are outlined in the subsequent sections (S.2-S.3).

\section{S.2. Heat Requirements and Raw Material Inputs for Various Cements}

The raw material requirements, intrinsic heat requirements, and amounts of $\mathrm{CO}_{2}$ emitted by the reactions involved in the production of each alternative cement were calculated using the four-step procedure described below.

\section{Step 1. Normalized cement phase compositions}

Typical phase compositions of each alternative cement were extracted from the literature (step 1a) and then normalized to 100 mass\% (step 1b). Small amounts (0.066-5\%) of solid admixtures (modeled here as gypsum) were included in these phase compositions, except for the CCSC, MOMC, and MOMS systems, where they were excluded.

\section{Step 2. Convert cement phase compositions into raw material requirements}

Cement phase compositions were specified into raw material equivalents through the following procedure:

a. Representing phases in terms of oxide constituents, e.g., $\mathrm{CaO}, \mathrm{SiO}_{2}, \mathrm{Al}_{2} \mathrm{O}_{3}$, etc.

b. Representing alternative cements in terms of bulk oxide compositions

c. Representing alternative cements in terms of raw materials

We converted bulk oxide compositions of cements into raw materials using typical inputs into cement clinker production, as well as equation $\mathrm{S} 1$ :

$$
\begin{aligned}
& a \mathrm{Al}_{2} \mathrm{Si}_{2} \mathrm{O}_{5}(\mathrm{OH})_{4}+b \mathrm{Al}(\mathrm{OH})_{3}+c \mathrm{MgCO}_{3}+\mathrm{dMg}_{2} \mathrm{SiO}_{4}+e \mathrm{SiO}_{2}+ \\
& \mathrm{fCaSO}_{4} \cdot 2 \mathrm{H}_{2} \mathrm{O}+\mathrm{gCaSO} \mathrm{CaFe}_{4}+h \mathrm{O}_{3}+i \mathrm{CaCO}_{3}+j \mathrm{CO}_{2}+k \mathrm{H}_{2} \mathrm{O} \longrightarrow \text { cement }
\end{aligned}
$$

where cement is specified in terms of moles of raw material inputs: $a$, kaolinite (clay); $b$, gibbsite (bauxite); $c$, magnesite; $d$, forsterite; $e$, quartz (siliceous rock, e.g., chert); $f$, gypsum; $g$, anhydrite; $h$, 
hematite (iron ore); $i$, calcite; $j, \mathrm{CO}_{2} ; k$, water. Positive values of coefficients $a$ - $k$ indicate raw material inputs to cement production, and negative values of coefficients $a-k$ indicate outputs of cement production. Kaolinite was preferentially specified as a raw material, with any remaining $\mathrm{Al}_{2} \mathrm{O}_{3}$ and $\mathrm{SiO}_{2}$ supplied by gibbsite and quartz, respectively.

Table S2 contains the oxide compositions of each clinker phase assessed.

\section{Step 3. Respecifying equation $S 1$ in terms of cement phase compositions}

The cement component in equation S1 (RHS) was respecified in terms of phase compositions, resulting in equation $(\mathrm{S} 2)$ :

$$
\begin{aligned}
& a \mathrm{Al}_{2} \mathrm{Si}_{2} \mathrm{O}_{5}(\mathrm{OH})_{4}+b \mathrm{Al}(\mathrm{OH})_{3}+c \mathrm{MgCO}_{3}+d \mathrm{Mg}_{2} \mathrm{SiO}_{4}+e \mathrm{SiO}_{2}+ \\
& f \mathrm{CaSO}_{4} \cdot 2 \mathrm{H}_{2} \mathrm{O}+g \mathrm{CaSO}_{4}+h \mathrm{Fe}_{2} \mathrm{O}_{3}+i \mathrm{CaCO}_{3}+j \mathrm{CO}_{2}+k \mathrm{H}_{2} \mathrm{O} \longrightarrow \\
& \mathrm{lCa}_{3} \mathrm{SiO}_{5}+\mathrm{mCa}_{2} \mathrm{SiO}_{4}+n \mathrm{Ca}_{3} \mathrm{Al}_{2} \mathrm{O}_{6}+o \mathrm{Ca}_{4} \mathrm{Al}_{2} \mathrm{Fe}_{2} \mathrm{O}_{10}+p \mathrm{CaO}+ \\
& \quad q \mathrm{CaSiO}_{3}+r \mathrm{Ca}_{4} \mathrm{Al}_{6} \mathrm{SO}_{16}+\mathrm{sCa}_{4} \mathrm{Al}_{6} \mathrm{SO}_{16}+t \mathrm{MgO}+u \mathrm{MgO}+v \mathrm{CaSO}_{4} \cdot 2 \mathrm{H}_{2} \mathrm{O}
\end{aligned}
$$

where the molar phase compositions of alternative cements are specified in terms of: $l$, alite; $m$, belite; $n$, aluminate; $o$, ferrite; $p$, free lime; $q$, wollastonite; $r$, ye'elimite derived from gypsum; $s$, ye'eliminite derived from anhydrite; $t$, periclase derived from magnesite; $u$, periclase derived from forsterite; and $v$, solid admixtures (modeled here as gypsum). Equation S2 is consistent with the environmental impact assessment system boundary used in this study (Figure 1).

Table S3 contains the pure raw mineral inputs necessary for the formation of each cement type considered.

\section{Step 4. Raw material derived enthalpy requirements and $\mathrm{CO}_{2}$ emissions}

Equation S2 was used to calculate raw material derived enthalpy requirements and $\mathrm{CO}_{2}$ emissions for cement production. Raw material derived $\mathrm{CO}_{2}$ emissions were obtained directly from parameter $i$. Raw material derived enthalpy requirements were obtained using standard state enthalpies of formation (the reference state is $298.15 \mathrm{~K}$ ) from ${ }^{1-3}$.

Our values of raw material derived enthalpy requirements and $\mathrm{CO}_{2}$ emissions generally differ by up to $30 \%$ from those reported in ${ }^{4}$, for the alternative cements analyzed in both studies. Here, we additionally include calcium sulfoaluminate-belite cement systems, as well as belite-ye'elimite-ferrite synthesized from gypsum rather than elemental sulfur, since the latter is not a typical input to cement production. The intrinsic heat requirements and $\mathrm{CO}_{2}$ emissions for the cements assessed in this work are presented in Table S4.

\section{S.3. Cradle-to-gate environmental impact assessment of cement binders}

To perform environmental impact assessments, an inventory of materials and processes was developed. Energy and material requirements from the calculations in Section S.2 were utilized with material 
resource purity, equipment efficiency, and transportation impacts to characterize the cradle-to-gate environmental impacts of producing each cement type. Sensitivity analyses were conducted to examine which parameters could lead to greatest variation in results. This work was conducted in four main steps.

\section{Step 1. Material inputs accounting for purity of mineral resources}

In Section S.2, raw material inputs to produce each cement system were quantified. However, these raw material resources are rarely mined pure. As such, greater masses of mineral resource inputs are required than that calculated in Section S.2 for actual production. Resource purities used here are shown in Table S5.

\section{Step 2. Unit processes}

To develop coherent environmental impact assessment results, unit processes were selected to minimize variability in regional representativeness. Specifically, production with California representative technologies was used. Unit processes for the production of raw material resources were from ${ }^{5}$ and are representative of United States acquisition / processing. Because the models developed assume production in California, kilns were modeled as having precalciner and preheater components, which is the predominant cement kiln technology present in the state ${ }^{6}$. Fuel type and quantity required for the kilns requirements were based on data from the United States Geological Survey (USGS) ${ }^{7}$; specifically, these values were based on the United States average demand for dry kilns. The energy required to produce $1 \mathrm{~kg}$ of $\mathrm{PC}$ clinker was estimated to be $3 \mathrm{MJ} / \mathrm{kg}$ based on ${ }^{8}$. Noting the manufacturing enthalpy for $\mathrm{PC}$ is less than half of this value, remaining energy required was assumed to be equipment inefficiency. Additional emissions of air pollutants from kiln processes were calculated based on the total emissions for precalciner/preheater dry kilns (data from ${ }^{9}$ ) minus air pollutant emissions calculated based on fuel type used (data from ${ }^{7}$ and emissions from stationary combustion of those fuels (data from ${ }^{10,11}$ ). The Californian electricity mix was selected, based on ${ }^{12}$. Transportation was modeled as by rail and was representative of United States rail transport ${ }^{5}$. We considered the environmental impacts of water emissions during cement production and for cement hydration to be negligible for the environmental impact categories assessed.

\section{Step 3. Inventory of materials, energy, transportation, and emissions}

Cradle-to-binder production inventories comprising raw material production, energy required for kilns and electricity demand during cement production, calcination (i.e., 'process-derived' emissions), transportation, and $\mathrm{CO}_{2}$ uptake (during curing of carbonatable cements), were compiled. Raw material inputs were calculated based on values presented in Table S3 and Table S5, accounting for their impurities. Energy requirements as presented in this work incorporate both kiln energy demand and electricity requirements in cement production. Kiln energy requirements were based on values presented in Table S4 with the additional energy inefficiency from the kilns. Based on the energy requirement modeled and the manufacturing enthalpy of PC, this inefficiency was considered to be $1.33 \mathrm{MJ} / \mathrm{kg}$ of clinker produced (44.4\% of the energy for the kiln). This level of inefficiency represents the kiln type and higher inefficiencies would be expected in older kilns. Electricity demand for cement production was based on Marceau et al. ${ }^{6}$ for precalciner/preheater dry kilns. Here, all raw materials were modeled as being transported $500 \mathrm{~km}$ from quarries to cement kilns. $\mathrm{CO}_{2}$ and water emissions are produced in the production of cement and were determined by the calculations performed in Section S.2. Carbonatable calcium silicate cements (CCSC) and the magnesium oxide cements (MOMS) were modeled as being cured with $\mathrm{CO}_{2}$, while the remaining cements were modeled as being cured with water. These curing 
processes require uptake of either $\mathrm{CO}_{2}$ or water, but since water was considered to have negligible contribution to the environmental impact categories assessed, only $\mathrm{CO}_{2}$ uptake in curing was captured in the inventory. $\mathrm{CO}_{2}$ uptake for the CCSC system was based on published values (from ${ }^{13}$ ) and uptake for the MOMS system was determined through stoichiometric balance $\left(\mathrm{MgO}+\mathrm{CO}_{2} \rightarrow \mathrm{MgCO} 3\right)$. To investigate each of these stages of the inventory in terms of their environmental impacts, stages were grouped into categories: raw material inputs (Table S6); energy demand (Table S7); transportation requirements (Table S8); $\mathrm{CO}_{2}$ emissions from chemical reactions during kilning (Table $\mathrm{S} 4$ ); $\mathrm{CO}_{2}$ uptake during curing (Table S9).

\section{Step 4. Environmental Impact Assessment}

Environmental impacts of the cradle-to-gate binder production inventories were assessed using the United States Environmental Protection Agency's TRACI method ${ }^{14}$. A breakdown of environmental impacts for each binder type in terms of stage of production (e.g., raw material production, kiln energy requirements) is presented in Tables S10-S14, and total impacts are shown in Table S15. A sensitivity analysis was conducted varying environmental impacts from each stage of production by $\pm 15 \%$. Furthermore, sensitivity to changes in sulfur and aluminum sources was considered, as was sensitivity to inefficiencies of the kiln, such that lower enthalpy processes continued to have the same energy inefficiency as current kilns. These sensitivity analysis results are presented in Figures S1-S10.

Since some of the cements presented in this work are not being produced at an industrial scale, the results from our study are inherently uncertain. We performed a sensitivity analysis to capture potential variation in our results based on ranges of sensitivity analyses used in other environmental impact assessments of novel cements, namely ${ }^{15,16}$. Results of these sensitivity analyses are presented in the main text for GHG emissions and, as noted, in Supplementary Figures S1-S10 for all impact categories considered. In future work, full Monte Carlo Simulations could be performed to more comprehensively account for uncertainty in the environmental impacts of alternative cement systems. This would be especially valuable once the cement systems start being used on the industrial scale, corresponding to more specific inputs and interpretations of parameter values. Further, considerations should be made for scenarios in which the degree of reduction for different environmental impact categories is not consistent across cement systems, that is, when certain systems result in greater reductions to one environmental impact category than another.

\section{References for this supplementary information file}

1. Lothenbach, B.; Matschei, T.; Möschner, G.; Glasser, F. P., Thermodynamic modelling of the effect of temperature on the hydration and porosity of Portland cement. Cement Concrete Res. 2008, 38 (1), 1-18.

2. $\quad$ Robie, R. A.; Hemingway, B. S. Thermodynamic Properties of Minerals and Related Substances at 298.15 K and 1 bar (105 Pascals) Pressure and at Higher Temperatures; Washington D.C., 1995.

3. Costa, U.; Massazza, F.; Testolin, M., Heats of formation of C4A3\$, 4SrO·3A12O3.SO3 and their mixed crystals. Il Cemento 1972, 2, 61-68.

4. $\quad$ Gartner, E.; Sui, T., Alternative cement clinkers. Cement Concrete Res. 2018, 114, 27-39.

5. $\quad$ LTS DATASMART LCI Package (US-EI SimaPro® Library).

https://ltsexperts.com/services/software/datasmart-life-cycle-inventory/ (accessed October 4, 2018).

6. Marceau, M. L.; Nisbet, M. A.; VanGeem, M. G. Life cycle inventory of Portland cement manufacture; Portland Cement Association: Skokie, Illinois, 2006.

7. $\quad$ van Oss, H. G. Minerals yearbook: cement 2015; United States Geological Survey: 2018; pp $16.1-16.33$ 
8. Habert, G.; Billard, C.; Rossi, P.; Chen, C.; Roussel, N., Cement production technology improvement compared to factor 4 objectives. Cement Concrete Res. 2010, 40 (5), 820-826.

9. USEPA Emission Factor Documentation for AP-42, Section 11.6: Portland Cement Manufacturing; 1994.

10. USEPA Air Emissions Inventories, Volume 2 Chapter 14: Uncontrolled Emission Factor Listing for Criteria Air Pollutants; 2001.

11. USEPA Locating and Estimating Air Emissions From Sources of Lead and Lead Compounds; 1998.

12. CEC Total System Electric Generation.

http://www.energy.ca.gov/almanac/electricity_data/total_system_power.html (accessed May 24, 2018).

13. Atakan, V.; Sahu, S.; Quinn, S.; Hu, X.; DeCristofaro, N. Why $\mathrm{CO}_{2}$ matters - advances in a new class of cement; Solidia Technologies, Reprint from ZKG International: Piscataway, New Jersey, USA, 2014.

14. USEPA Tool for Reduction and Assessment of Chemicals and Other Environmental Impacts (TRACI). https://19january2017snapshot.epa.gov/chemical-research/tool-reduction-and-assessmentchemicals-and-other-environmental-impacts-traci_html (accessed December 31, 2018).

15. Hanein, T.; Galvez-Martos, J.-L.; Bannerman, M. N., Carbon footprint of calcium sulfoaluminate clinker production. J. Clean. Prod. 2018, 172, 2278-2287.

16. Ruan, S.; Unluer, C., Comparative life cycle assessment of reactive $\mathrm{MgO}$ and Portland cement production. J. Clean. Prod. 2016, 137, 258-273.

17. Chen, I. A.; Hargis, C. W.; Juenger, M. C. G., Understanding expansion in calcium sulfoaluminate-belite cements. Cement Concrete Res. 2012, 42 (1), 51-60.

18. Boughanmia, S.; labidia, I.; Tissb, H.; Megrichea, A., The effect of marl and clay compositions on the Portland cement quality. J. Tunisian Chem. S. 2016, (18), 43-51.

19. Cañaveras, J. C.; Sánchez-Moral, S.; Sanz-Rubio, E.; Hoyos, M., Meteoric calcitization of magnesite in Miocene lacustrine deposits (Calatayud basin, NE Spain). Sediment. Geol. 1998, 119 (3), 183-194.

20. Birch, R. E.; Harvey, F. A., Forsterite and Other Magnesium Silicates as Refractories. 1935, 18 (1 - 12), 176-192.

21. Götze, J., Classification, Mineralogy and Industrial Potential of SiO2 Minerals and Rocks. In Quartz: Deposits, Mineralogy and Analytics, Jens Götze, R. M., Ed. Springer: 2012.

22. Harrison, D. J., High-purity limestones in England and Wales. 1993, 26 (4), 293-303.

23. Frost, R. L., Bauxite. In Encyclopedia of Sediments and Sedimentary Rocks, Middleton, G. V.; Church, M. J.; Coniglio, M.; Hardie, L. A.; Longstaffe, F. J., Eds. Springer Netherlands: Dordrecht, 2003; pp 48-51. 
Table S1. Notation style

\begin{tabular}{|l|l|}
\hline Abbreviation & Definition \\
\hline C & $\mathrm{CaO}$ \\
\hline S & $\mathrm{SiO}_{2}$ \\
\hline A & $\mathrm{Al}_{2} \mathrm{O}_{3}$ \\
\hline F & $\mathrm{Fe}_{2} \mathrm{O}_{3}$ \\
\hline $\mathrm{H}$ & $\mathrm{H}_{2} \mathrm{O}$ \\
\hline$\$$ & $\mathrm{SO}_{3}$ \\
\hline
\end{tabular}

Table S2. Oxide compositions of clinker phases.

\begin{tabular}{|l|c|c|c|c|c|c|c|}
\hline Oxide $(\mathrm{g} / 1 \mathrm{~g}$ phase) & $\mathrm{CaO}$ & $\mathrm{SiO}_{2}$ & $\mathrm{Al}_{2} \mathrm{O}_{3}$ & $\mathrm{Fe}_{2} \mathrm{O}_{3}$ & $\mathrm{SO}_{3}$ & $\mathrm{CO}_{2}$ & $\mathrm{MgO}$ \\
\hline Alite, $\left(\mathrm{C}_{3} \mathrm{~S}\right)$ & 0.737 & 0.263 & 0 & 0 & 0 & 0 & 0 \\
\hline Belite, $\left(\mathrm{C}_{2} \mathrm{~S}\right)$ & 0.651 & 0.349 & 0 & 0 & 0 & 0 & 0 \\
\hline Aluminate, $\left(\mathrm{C}_{3} \mathrm{~A}\right)$ & 0.622 & 0 & 0.378 & 0 & 0 & 0 & 0 \\
\hline Ferrite, $\left(\mathrm{C}_{4} \mathrm{AF}\right)$ & 0.461 & 0 & 0.210 & 0.329 & 0 & 0 & 0 \\
\hline Quicklime, $(\mathrm{C})$ & 1 & 0 & 0 & 0 & 0 & 0 & 0 \\
\hline Wollastonite, $(\mathrm{CS})$ & 0.483 & 0.517 & 0 & 0 & 0 & 0 & 0 \\
\hline Ye'elimite $\left(\mathrm{C}_{4} \mathrm{~A}_{3} \$\right.$ ) [from $\left.\mathrm{CaSO}_{4} .2 \mathrm{H}_{2} \mathrm{O}\right]$ & 0.367 & 0 & 0.502 & 0 & 0.131 & 0 & 0 \\
\hline Ye'elimite $\left(\mathrm{C}_{4} \mathrm{~A}_{3} \$\right.$ ) [from $\left.\mathrm{CaSO}_{4}\right]$ & 0.367 & 0 & 0.502 & 0 & 0.131 & 0 & 0 \\
\hline Periclase $(\mathrm{MgO})$ from $\mathrm{MgCO}_{3}$ ] & 0 & 0 & 0 & 0 & 0 & 0 & 1 \\
\hline Periclase $(\mathrm{MgO})\left[\right.$ from $\mathrm{Mg}_{2} \mathrm{SiO}_{4}$ ] & 0 & 0 & 0 & 0 & 0 & 0 & 1 \\
\hline other (modeled as gypsum) & 0.326 & 0 & 0 & 0 & 0.465 & 0 & 0 \\
\hline
\end{tabular}

Table S3. Pure raw mineral inputs needed for cement (in units of $\mathrm{g}$ raw material per $\mathrm{g}$ cement).

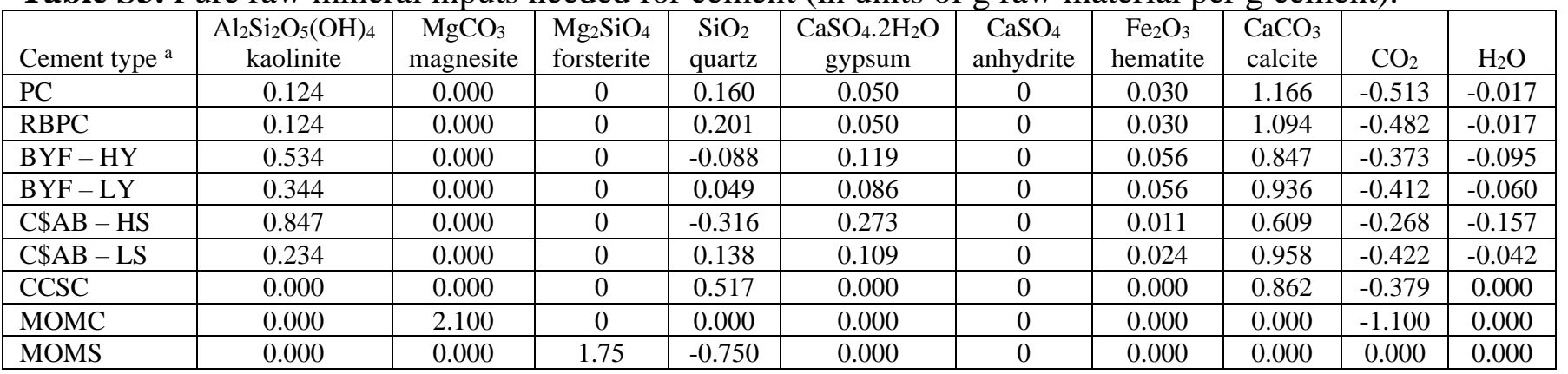

${ }^{\mathrm{a}}$ Based on clinker phase composition from Gartner and Sui ${ }^{4}$ and from Chen et al. ${ }^{17}$.

Table S4. Intrinsic heat requirements and $\mathrm{CO}_{2}$ emissions of reactions required for each cement type considered.

\begin{tabular}{|l|l|l|}
\hline Cement type & Intrinsic heat requirement of reaction $(\mathrm{kJ} / 1 \mathrm{~g}$ cement $)$ & Intrinsic $\mathrm{CO}_{2}$ emissions of reaction $(\mathrm{g} / 1 \mathrm{~g}$ cement $)$ \\
\hline PC & 1.67 & 0.513 \\
\hline RBPC & 1.43 & 0.482 \\
\hline BYF - HY & 1.25 & 0.373 \\
\hline BYF - LY & 1.27 & 0.412 \\
\hline C $\$ A B-H S$ & 1.06 & 0.268 \\
\hline C \$AB - LS & 1.22 & 0.422 \\
\hline CCSC & 0.766 & 0.379 \\
\hline MOMC & 2.93 & 1.10 \\
\hline MOMS & 0.739 & 0 \\
\hline
\end{tabular}


Table S5. Purity of mineral resources.

\begin{tabular}{|l|l|l|}
\hline Mineral resource & \% Composition of raw material & Reference \\
\hline $\mathrm{Al}_{2} \mathrm{Si}_{2} \mathrm{O}_{5}(\mathrm{OH})_{4}$ & 61.3 & 18 \\
\hline $\mathrm{MgCO}_{3}$ & 93.7 & 19 \\
\hline $\mathrm{Mg}_{2} \mathrm{SiO}_{4}$ & 88.4 & 20 \\
\hline $\mathrm{SiO}_{2}$ & 99.9 & 21 \\
\hline $\mathrm{CaSO}_{4} 2 \mathrm{H}_{2} \mathrm{O}$ & 95 & Estimate \\
\hline $\mathrm{CaSO}_{4}$ & 95 & Estimate \\
\hline $\mathrm{Fe}_{2} \mathrm{O}_{3}$ & 95 & Based on unit process model \\
\hline $\mathrm{CaCO}_{3}$ & 93.5 & 22 \\
\hline $\mathrm{Al}(\mathrm{OH})_{3}$ & 95 & 23 \\
\hline
\end{tabular}

Table S6. Raw material inventory.

\begin{tabular}{|l|c|c|c|c|c|c|}
\hline Cement type & limestone, $\mathrm{kg} / \mathrm{kg}$ & clay, $\mathrm{kg} / \mathrm{kg}$ & iron ore, $\mathrm{kg} / \mathrm{kg}$ & anhydrite, $\mathrm{kg} / \mathrm{kg}$ & silica sand, $\mathrm{kg} / \mathrm{kg}$ & $\mathrm{gypsum}, \mathrm{kg} / \mathrm{kg}$ \\
\hline OPC & 1.247 & 0.203 & 0.031 & 0.053 & 0.160 & 0.053 \\
\hline RBPC & 1.171 & 0.203 & 0.031 & 0.053 & 0.201 & 0.053 \\
\hline BYF - HY & 0.906 & 0.872 & 0.059 & 0.125 & -0.088 \\
\hline BYF - LY & 1.001 & 0.561 & 0.059 & 0.091 & 0.049 & 0.125 \\
\hline C\$AB - HS & 0.652 & 1.382 & 0.011 & 0.288 & -0.316 & 0.091 \\
\hline C \$AB - LS & 1.025 & 0.381 & 0.025 & 0.115 & 0.138 & 0.288 \\
\hline CCSC & 0.922 & 0 & 0 & 0 & 0.518 & 0 \\
\hline MOMC & 0 & 2.241 & 0 & 0 & 0 & 0 \\
\hline MOMS & 0 & 1.980 & 0 & 0 & -0.751 & 0 \\
\hline
\end{tabular}

Table S7. Energy inventory and air pollutant emissions (excluding $\mathrm{CO}_{2}$ emissions noted in Table S3).

\begin{tabular}{|c|c|c|c|c|c|c|c|}
\hline Cement type & $\begin{array}{l}\text { Kiln thermal } \\
\text { energy } \\
\text { (enthalpy), } \\
\text { fraction of } 3 \\
\mathrm{MJ} / \mathrm{kg}\end{array}$ & $\begin{array}{l}\text { Kiln thermal } \\
\text { energy } \\
\text { (inefficiency), } \\
\text { fraction of } 3 \\
\mathrm{MJ} / \mathrm{kg}\end{array}$ & $\begin{array}{c}\text { Kiln } \mathrm{NO}_{\mathrm{X}} \\
\text { emissions } \\
\text { (process- } \\
\text { based), } \mathrm{kg} / \mathrm{kg}\end{array}$ & $\begin{array}{c}\text { Kiln } \mathrm{SO}_{\mathrm{x}} \\
\text { emissions } \\
\text { (process- } \\
\text { based), } \mathrm{kg} / \mathrm{kg}\end{array}$ & $\begin{array}{c}\text { Kiln CO } \\
\text { emissions } \\
\text { (process- } \\
\text { based), } \mathrm{kg} / \mathrm{kg}\end{array}$ & $\begin{array}{c}\text { Kiln } \mathrm{Pb} \\
\text { emissions } \\
\text { (process- } \\
\text { based), } \mathrm{kg} / \mathrm{kg}\end{array}$ & $\begin{array}{c}\text { Electricity, } \\
\mathrm{MJ} / \mathrm{kg}\end{array}$ \\
\hline OPC & 0.556 & 0.444 & $1.83 \mathrm{E}-03$ & $4.50 \mathrm{E}-05$ & $1.76 \mathrm{E}-03$ & $6.00 \mathrm{E}-05$ & 0.517 \\
\hline RBPC & 0.477 & 0.444 & $1.83 \mathrm{E}-03$ & $4.50 \mathrm{E}-05$ & $1.76 \mathrm{E}-03$ & $6.00 \mathrm{E}-05$ & 0.517 \\
\hline $\mathrm{BYF}-\mathrm{HY}$ & 0.415 & 0.444 & $1.83 \mathrm{E}-03$ & $4.50 \mathrm{E}-05$ & $1.76 \mathrm{E}-03$ & $6.00 \mathrm{E}-05$ & 0.517 \\
\hline $\mathrm{C} \$ \mathrm{AB}-\mathrm{LS}$ & 0.405 & 0.444 & $1.83 \mathrm{E}-03$ & $4.50 \mathrm{E}-05$ & $1.76 \mathrm{E}-03$ & $6.00 \mathrm{E}-05$ & 0.517 \\
\hline CCSC & 0.255 & 0.444 & $1.83 \mathrm{E}-03$ & $4.50 \mathrm{E}-05$ & $1.76 \mathrm{E}-03$ & $6.00 \mathrm{E}-05$ & 0.517 \\
\hline MOMC & 0.978 & 0.444 & $1.83 \mathrm{E}-03$ & $4.50 \mathrm{E}-05$ & $1.76 \mathrm{E}-03$ & $6.00 \mathrm{E}-05$ & 0.517 \\
\hline MOMS & 0.246 & 0.444 & $1.83 \mathrm{E}-03$ & $4.50 \mathrm{E}-05$ & $1.76 \mathrm{E}-03$ & $6.00 \mathrm{E}-05$ & 0.517 \\
\hline
\end{tabular}


Table S8. Transportation inventory.

\begin{tabular}{|l|c|}
\hline Cement type & Transportation by rail (t.km) \\
\hline OPC & 0.874 \\
\hline RBPC & 0.855 \\
\hline BYF - HY & 0.999 \\
\hline BYF - LY & 0.926 \\
\hline C \$AB - HS & 1.152 \\
\hline C \$AB - LS & 0.900 \\
\hline CCSC & 0.720 \\
\hline MOMC & 1.121 \\
\hline MOMS & 0.614 \\
\hline
\end{tabular}

Table S9. $\mathrm{CO}_{2}$ uptake during curing.

\begin{tabular}{|l|c|}
\hline Cement type & $\mathrm{CO}_{2}, \mathrm{~kg} / \mathrm{kg}$ \\
\hline OPC & 0 \\
\hline RBPC & 0 \\
\hline BYF - HY & 0 \\
\hline BYF - LY & 0 \\
\hline C \$AB - HS & 0 \\
\hline C \$AB - LS & 0 \\
\hline CCSC & 0.216 \\
\hline MOMC & 0.524 \\
\hline MOMS & 0.524 \\
\hline
\end{tabular}

Table S10. Environmental impacts from the raw material life cycle stage (impacts are specified per $\mathrm{kg}$ of cement).

\begin{tabular}{|c|c|c|c|c|c|c|c|c|c|c|}
\hline Cement type & $\begin{array}{c}\text { Ozone } \\
\text { depletion, } \\
\text { kg CFC-11 } \\
\text { eq / kg } \\
\end{array}$ & $\begin{array}{c}\text { Global } \\
\text { warming, } \\
\mathrm{kg} \mathrm{CO} 2 \\
\mathrm{eq} / \mathrm{kg}\end{array}$ & $\begin{array}{l}\text { Smog, kg } \\
\mathrm{O}_{3} \mathrm{eq} / \mathrm{kg}\end{array}$ & $\begin{array}{c}\text { Acidification, } \\
\mathrm{kg} \mathrm{SO}_{2} \text { eq / } \\
\mathrm{kg}\end{array}$ & $\begin{array}{l}\text { Eutrophication, } \\
\mathrm{kg} \mathrm{N} \text { eq / kg }\end{array}$ & $\begin{array}{c}\text { Carcinogenics, } \\
\text { CTUh / kg }\end{array}$ & $\begin{array}{c}\text { Non } \\
\text { carcinogenics, } \\
\text { CTUh / kg } \\
\end{array}$ & $\begin{array}{c}\text { Respiratory } \\
\text { effects, kg } \\
\text { PM2.5 eq / } \\
\text { kg } \\
\end{array}$ & $\begin{array}{c}\text { Ecotoxicity, } \\
\text { CTUe / kg }\end{array}$ & $\begin{array}{c}\text { Fossil fuel } \\
\text { depletion, } \\
\text { MJ surplus } \\
\text { / kg }\end{array}$ \\
\hline $\mathrm{OPC}$ & $1.01 \mathrm{E}-09$ & $1.25 \mathrm{E}-02$ & $1.02 \mathrm{E}-03$ & $6.16 \mathrm{E}-05$ & $2.12 \mathrm{E}-05$ & $4.72 \mathrm{E}-10$ & $2.31 \mathrm{E}-09$ & $4.50 \mathrm{E}-05$ & $4.59 \mathrm{E}-02$ & $1.86 \mathrm{E}-02$ \\
\hline RBPC & $1.16 \mathrm{E}-09$ & $1.30 \mathrm{E}-02$ & $1.04 \mathrm{E}-03$ & $6.25 \mathrm{E}-05$ & $2.09 \mathrm{E}-05$ & $4.72 \mathrm{E}-10$ & $2.36 \mathrm{E}-09$ & $4.52 \mathrm{E}-05$ & $4.51 \mathrm{E}-02$ & $1.98 \mathrm{E}-02$ \\
\hline BYF - HY & $3.70 \mathrm{E}-10$ & $7.78 \mathrm{E}-03$ & $1.55 \mathrm{E}-03$ & $6.96 \mathrm{E}-05$ & $1.76 \mathrm{E}-05$ & $3.79 \mathrm{E}-10$ & $1.29 \mathrm{E}-09$ & $9.19 \mathrm{E}-05$ & $3.43 \mathrm{E}-02$ & $1.06 \mathrm{E}-02$ \\
\hline BYF - LY & $7.65 \mathrm{E}-10$ & $1.04 \mathrm{E}-02$ & $1.39 \mathrm{E}-03$ & $6.93 \mathrm{E}-05$ & $1.96 \mathrm{E}-05$ & $4.36 \mathrm{E}-10$ & $1.84 \mathrm{E}-09$ & $7.96 \mathrm{E}-05$ & $4.03 \mathrm{E}-02$ & $1.52 \mathrm{E}-02$ \\
\hline $\mathrm{C} \$ \mathrm{AB}-\mathrm{HS}$ & $-3.08 \mathrm{E}-10$ & $2.40 \mathrm{E}-03$ & $1.77 \mathrm{E}-03$ & $6.43 \mathrm{E}-05$ & $1.12 \mathrm{E}-05$ & $2.11 \mathrm{E}-10$ & $4.77 \mathrm{E}-11$ & $1.14 \mathrm{E}-04$ & $1.63 \mathrm{E}-02$ & $1.89 \mathrm{E}-03$ \\
\hline $\mathrm{C} \$ \mathrm{AB}-\mathrm{LS}$ & $1.00 \mathrm{E}-09$ & $1.15 \mathrm{E}-02$ & $1.19 \mathrm{E}-03$ & $6.38 \mathrm{E}-05$ & $1.90 \mathrm{E}-05$ & $4.25 \mathrm{E}-10$ & $1.98 \mathrm{E}-09$ & $6.27 \mathrm{E}-05$ & $3.92 \mathrm{E}-02$ & $1.75 \mathrm{E}-02$ \\
\hline CCSC & $2.21 \mathrm{E}-09$ & $1.73 \mathrm{E}-02$ & $8.28 \mathrm{E}-04$ & $5.86 \mathrm{E}-05$ & $2.01 \mathrm{E}-05$ & $4.90 \mathrm{E}-10$ & $2.96 \mathrm{E}-09$ & $4.03 \mathrm{E}-06$ & 4.30E-02 & $2.96 \mathrm{E}-02$ \\
\hline MOMC & 1.07E-09 & $6.63 \mathrm{E}-03$ & $1.92 \mathrm{E}-03$ & $6.41 \mathrm{E}-05$ & $7.53 \mathrm{E}-06$ & $1.93 \mathrm{E}-10$ & $2.77 \mathrm{E}-10$ & $8.43 \mathrm{E}-06$ & $5.03 \mathrm{E}-03$ & $1.36 \mathrm{E}-02$ \\
\hline MOMS & $-2.06 \mathrm{E}-09$ & $-1.13 \mathrm{E}-02$ & $8.83 \mathrm{E}-04$ & $5.21 \mathrm{E}-06$ & $-6.22 \mathrm{E}-06$ & $-2.03 \mathrm{E}-10$ & $-2.42 \mathrm{E}-09$ & $3.29 \mathrm{E}-06$ & $-2.00 \mathrm{E}-02$ & $-2.12 \mathrm{E}-02$ \\
\hline
\end{tabular}


Table S11. Environmental impacts from energy inputs (impacts are per $\mathrm{kg}$ of cement).

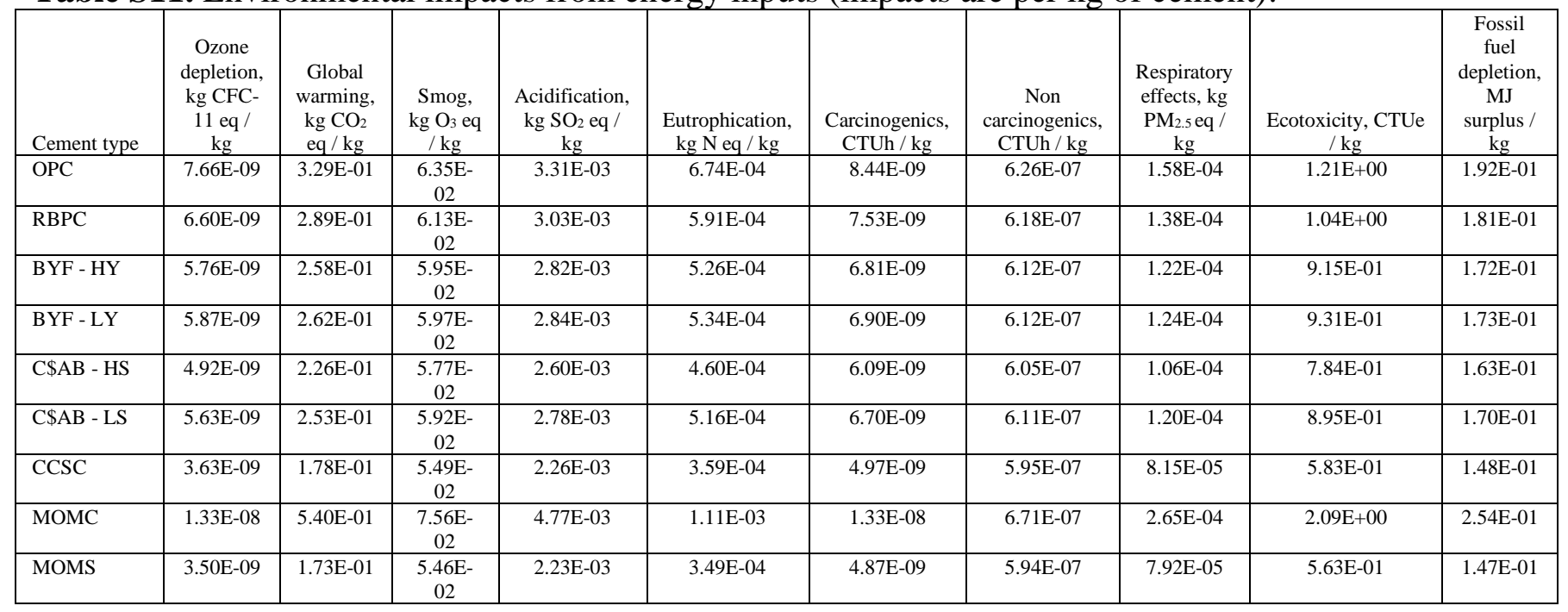

Table S12. Environmental impacts from transportation inputs (impacts are per kg of cement).

\begin{tabular}{|c|c|c|c|c|c|c|c|c|c|c|}
\hline Cement type & $\begin{array}{c}\text { Ozone } \\
\text { depletion, } \\
\text { kg CFC-11 } \\
\text { eq / kg }\end{array}$ & $\begin{array}{c}\text { Global } \\
\text { warming, } \\
\mathrm{kg} \mathrm{CO}_{2} \\
\text { eq } / \mathrm{kg}\end{array}$ & $\begin{array}{l}\text { Smog, kg } \\
\mathrm{O}_{3} \text { eq / kg }\end{array}$ & $\begin{array}{l}\text { Acidification, } \\
\mathrm{kg} \mathrm{SO}_{2} \text { eq / } \\
\mathrm{kg}\end{array}$ & $\begin{array}{l}\text { Eutrophication, } \\
\mathrm{kg} \mathrm{N} \text { eq } / \mathrm{kg}\end{array}$ & $\begin{array}{c}\text { Carcinogenics, } \\
\text { CTUh / kg }\end{array}$ & $\begin{array}{c}\text { Non } \\
\text { carcinogenics, } \\
\text { CTUh / kg }\end{array}$ & $\begin{array}{c}\text { Respiratory } \\
\text { effects, kg } \\
\mathrm{PM}_{2.5} \text { eq / } \\
\text { kg }\end{array}$ & $\begin{array}{l}\text { Ecotoxicity, } \\
\text { CTUe / kg }\end{array}$ & $\begin{array}{c}\text { Fossil fuel } \\
\text { depletion, } \\
\text { MJ surplus } \\
\text { / kg }\end{array}$ \\
\hline OPC & $6.54 \mathrm{E}-09$ & $4.60 \mathrm{E}-02$ & $1.38 \mathrm{E}-02$ & $4.63 \mathrm{E}-04$ & $6.97 \mathrm{E}-05$ & $4.26 \mathrm{E}-09$ & $5.90 \mathrm{E}-09$ & $2.86 \mathrm{E}-05$ & $1.19 \mathrm{E}-01$ & $8.51 \mathrm{E}-02$ \\
\hline RBPC & $6.41 \mathrm{E}-09$ & $4.51 \mathrm{E}-02$ & $1.35 \mathrm{E}-02$ & $4.54 \mathrm{E}-04$ & $6.82 \mathrm{E}-05$ & $4.17 \mathrm{E}-09$ & $5.78 \mathrm{E}-09$ & $2.80 \mathrm{E}-05$ & $1.16 \mathrm{E}-01$ & $8.33 \mathrm{E}-02$ \\
\hline BYF - HY & 7.49E-09 & $5.26 \mathrm{E}-02$ & $1.58 \mathrm{E}-02$ & $5.30 \mathrm{E}-04$ & 7.97E-05 & $4.87 \mathrm{E}-09$ & $6.75 \mathrm{E}-09$ & $3.27 \mathrm{E}-05$ & $1.36 \mathrm{E}-01$ & $9.73 \mathrm{E}-02$ \\
\hline BYF - LY & $6.94 \mathrm{E}-09$ & $4.88 \mathrm{E}-02$ & $1.46 \mathrm{E}-02$ & $4.91 \mathrm{E}-04$ & $7.39 \mathrm{E}-05$ & $4.51 \mathrm{E}-09$ & $6.26 \mathrm{E}-09$ & $3.03 \mathrm{E}-05$ & $1.26 \mathrm{E}-01$ & $9.02 \mathrm{E}-02$ \\
\hline $\mathrm{C} \$ \mathrm{AB}-\mathrm{HS}$ & $8.63 \mathrm{E}-09$ & $6.07 \mathrm{E}-02$ & $1.82 \mathrm{E}-02$ & $6.11 \mathrm{E}-04$ & $9.19 \mathrm{E}-05$ & $5.62 \mathrm{E}-09$ & 7.79E-09 & $3.77 \mathrm{E}-05$ & $1.57 \mathrm{E}-01$ & $1.12 \mathrm{E}-01$ \\
\hline $\mathrm{C} \$ \mathrm{AB}-\mathrm{LS}$ & $6.74 \mathrm{E}-09$ & $4.74 \mathrm{E}-02$ & $1.42 \mathrm{E}-02$ & $4.77 \mathrm{E}-04$ & $7.18 \mathrm{E}-05$ & $4.39 \mathrm{E}-09$ & $6.08 \mathrm{E}-09$ & $2.95 \mathrm{E}-05$ & $1.22 \mathrm{E}-01$ & $8.76 \mathrm{E}-02$ \\
\hline $\mathrm{CCSC}$ & $5.39 \mathrm{E}-09$ & $3.79 \mathrm{E}-02$ & $1.14 \mathrm{E}-02$ & $3.82 \mathrm{E}-04$ & $5.74 \mathrm{E}-05$ & $3.51 \mathrm{E}-09$ & $4.86 \mathrm{E}-09$ & $2.36 \mathrm{E}-05$ & $9.78 \mathrm{E}-02$ & $7.01 \mathrm{E}-02$ \\
\hline MOMC & $8.40 \mathrm{E}-09$ & $5.90 \mathrm{E}-02$ & $1.77 \mathrm{E}-02$ & $5.94 \mathrm{E}-04$ & $8.94 \mathrm{E}-05$ & 5.46E-09 & $\begin{array}{l}7.57 \mathrm{E}-09 \\
\end{array}$ & $3.67 \mathrm{E}-05$ & $1.52 \mathrm{E}-01$ & $1.09 \mathrm{E}-01$ \\
\hline MOMS & $4.60 \mathrm{E}-09$ & $3.24 \mathrm{E}-02$ & $9.70 \mathrm{E}-03$ & $3.26 \mathrm{E}-04$ & $4.90 \mathrm{E}-05$ & $3.00 \mathrm{E}-09$ & $4.15 \mathrm{E}-09$ & $2.01 \mathrm{E}-05$ & $8.35 \mathrm{E}-02$ & $5.98 \mathrm{E}-02$ \\
\hline
\end{tabular}

Table S13. Environmental impacts from calcination $-\mathrm{CO}_{2}$ emissions during kilning (impacts are per kg of cement).

\begin{tabular}{|c|c|c|c|c|c|c|c|c|c|c|}
\hline Cement type & $\begin{array}{c}\text { Ozone } \\
\text { depletion, } \\
\text { kg CFC- } \\
11 \mathrm{eq} / \mathrm{kg}\end{array}$ & $\begin{array}{c}\text { Global } \\
\text { warming, kg } \\
\mathrm{CO}_{2} \mathrm{eq} / \mathrm{kg}\end{array}$ & $\begin{array}{l}\mathrm{Smog} \\
\mathrm{kg} \mathrm{O}_{3} \\
\mathrm{eq} / \mathrm{kg}\end{array}$ & $\begin{array}{c}\text { Acidification, } \\
\mathrm{kg} \mathrm{SO}_{2} \text { eq / } \\
\mathrm{kg}\end{array}$ & $\begin{array}{c}\text { Eutrophication, } \\
\mathrm{kg} \mathrm{N} \text { eq / kg }\end{array}$ & $\begin{array}{c}\text { Carcinogenics, } \\
\text { CTUh / kg }\end{array}$ & $\begin{array}{c}\text { Non } \\
\text { carcinogenics, } \\
\text { CTUh / kg }\end{array}$ & $\begin{array}{c}\text { Respiratory } \\
\text { effects, kg } \\
\mathrm{PM}_{2.5} \text { eq / } \\
\mathrm{kg}\end{array}$ & $\begin{array}{c}\text { Ecotoxicity, } \\
\text { CTUe / kg }\end{array}$ & $\begin{array}{c}\text { Fossil fue } \\
\text { depletion, } \\
\text { MJ } \\
\text { surplus / } \\
\text { kg }\end{array}$ \\
\hline OPC & 0 & $5.13 \mathrm{E}-01$ & 0 & 0 & 0 & 0 & 0 & 0 & 0 & 0 \\
\hline RBPC & 0 & $4.82 \mathrm{E}-01$ & 0 & 0 & 0 & 0 & 0 & 0 & 0 & 0 \\
\hline BYF - HY & 0 & $3.73 \mathrm{E}-01$ & 0 & 0 & 0 & 0 & 0 & 0 & 0 & 0 \\
\hline BYF - LY & 0 & $4.12 \mathrm{E}-01$ & 0 & 0 & 0 & 0 & 0 & 0 & 0 & 0 \\
\hline $\mathrm{C} \$ \mathrm{AB}-\mathrm{HS}$ & 0 & $2.68 \mathrm{E}-01$ & 0 & 0 & 0 & 0 & 0 & 0 & 0 & 0 \\
\hline $\mathrm{C} \$ \mathrm{AB}-\mathrm{LS}$ & 0 & 4.22E-01 & 0 & 0 & 0 & 0 & 0 & 0 & 0 & 0 \\
\hline CCSC & 0 & $3.79 \mathrm{E}-01$ & 0 & 0 & 0 & 0 & 0 & 0 & 0 & 0 \\
\hline MOMC & 0 & $1.10 \mathrm{E}+00$ & 0 & 0 & 0 & 0 & 0 & 0 & 0 & 0 \\
\hline MOMS & 0 & 0 & 0 & 0 & 0 & 0 & 0 & 0 & 0 & 0 \\
\hline
\end{tabular}


Table S14. Environmental impacts from curing (impacts are per kg of cement).

\begin{tabular}{|c|c|c|c|c|c|c|c|c|c|c|}
\hline Cement type & $\begin{array}{c}\text { Ozone } \\
\text { depletion, } \\
\mathrm{kg} \mathrm{CFC}-11 \\
\mathrm{eq} / \mathrm{kg}\end{array}$ & $\begin{array}{c}\text { Global } \\
\text { warming, } \\
\mathrm{kg} \mathrm{CO} 2 \\
\mathrm{eq} / \mathrm{kg}\end{array}$ & $\begin{array}{l}\text { Smog, kg } \\
\mathrm{O}_{3} \mathrm{eq} / \mathrm{kg}\end{array}$ & $\begin{array}{c}\text { Acidification, } \\
\mathrm{kg} \mathrm{SO}_{2} \text { eq / } \\
\mathrm{kg}\end{array}$ & $\begin{array}{c}\text { Eutrophication, } \\
\mathrm{kg} \mathrm{N} \mathrm{eq} \mathrm{/} \mathrm{kg}\end{array}$ & $\begin{array}{c}\text { Carcinogenics, } \\
\text { CTUh / kg }\end{array}$ & $\begin{array}{c}\text { Non } \\
\text { carcinogenics, } \\
\text { CTUh / kg }\end{array}$ & $\begin{array}{c}\text { Respiratory } \\
\text { effects, kg } \\
\mathrm{PM}_{2.5} \mathrm{eq} / \\
\mathrm{kg}\end{array}$ & $\begin{array}{c}\text { Ecotoxicity, } \\
\text { CTUe / kg }\end{array}$ & $\begin{array}{c}\text { Fossil fuel } \\
\text { depletion, } \\
\text { MJ surplus } \\
\text { / kg }\end{array}$ \\
\hline OPC & 0 & 0 & 0 & 0 & 0 & 0 & 0 & 0 & 0 & 0 \\
\hline RBPC & 0 & 0 & 0 & 0 & 0 & 0 & 0 & 0 & 0 & 0 \\
\hline BYF - HY & 0 & 0 & 0 & 0 & 0 & 0 & 0 & 0 & 0 & 0 \\
\hline BYF - LY & 0 & 0 & 0 & 0 & 0 & 0 & 0 & 0 & 0 & 0 \\
\hline $\mathrm{C} \$ \mathrm{AB}-\mathrm{HS}$ & 0 & 0 & 0 & 0 & 0 & 0 & 0 & 0 & 0 & 0 \\
\hline $\mathrm{C} \$ \mathrm{AB}-\mathrm{LS}$ & 0 & 0 & 0 & 0 & 0 & 0 & 0 & 0 & 0 & 0 \\
\hline CCSC & 0 & -0.22 & 0 & 0 & 0 & 0 & 0 & 0 & 0 & 0 \\
\hline MOMC & 0 & -0.52 & 0 & 0 & 0 & 0 & 0 & 0 & 0 & 0 \\
\hline MOMS & 0 & -0.52 & 0 & 0 & 0 & 0 & 0 & 0 & 0 & 0 \\
\hline
\end{tabular}

Table S15. Total environmental impacts (impacts are per kg of cement).

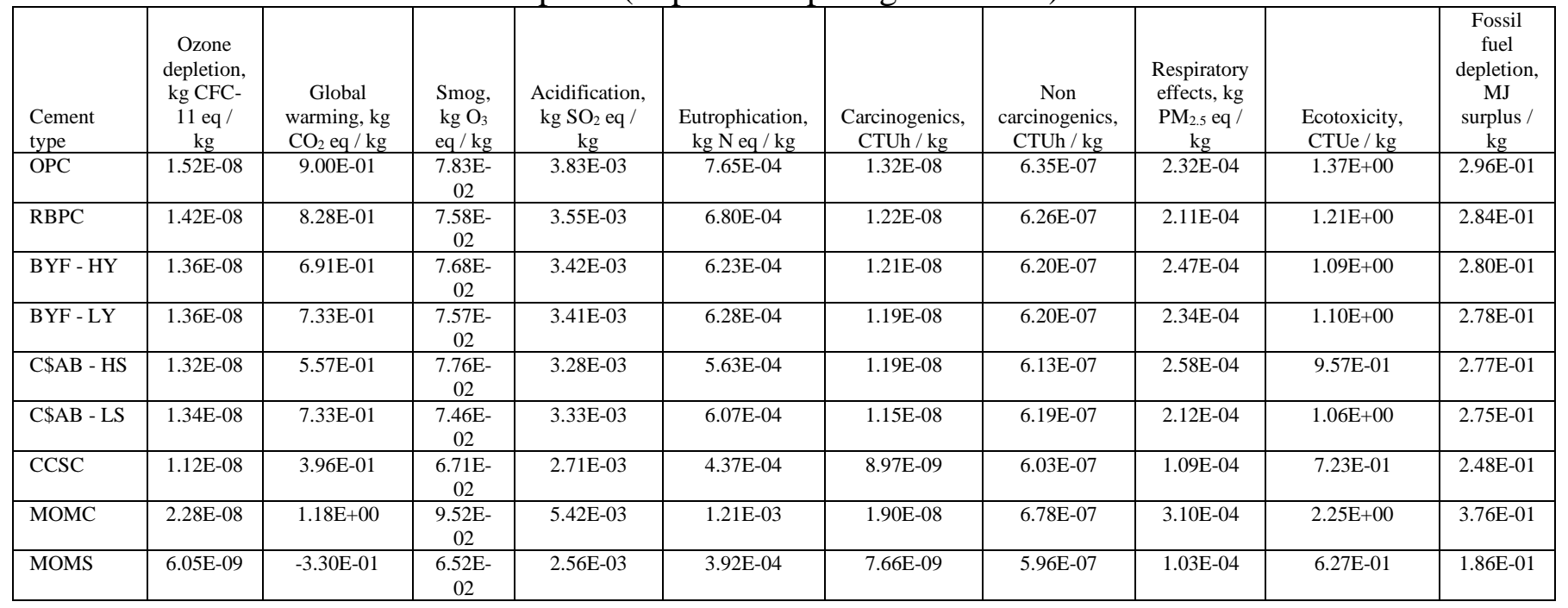




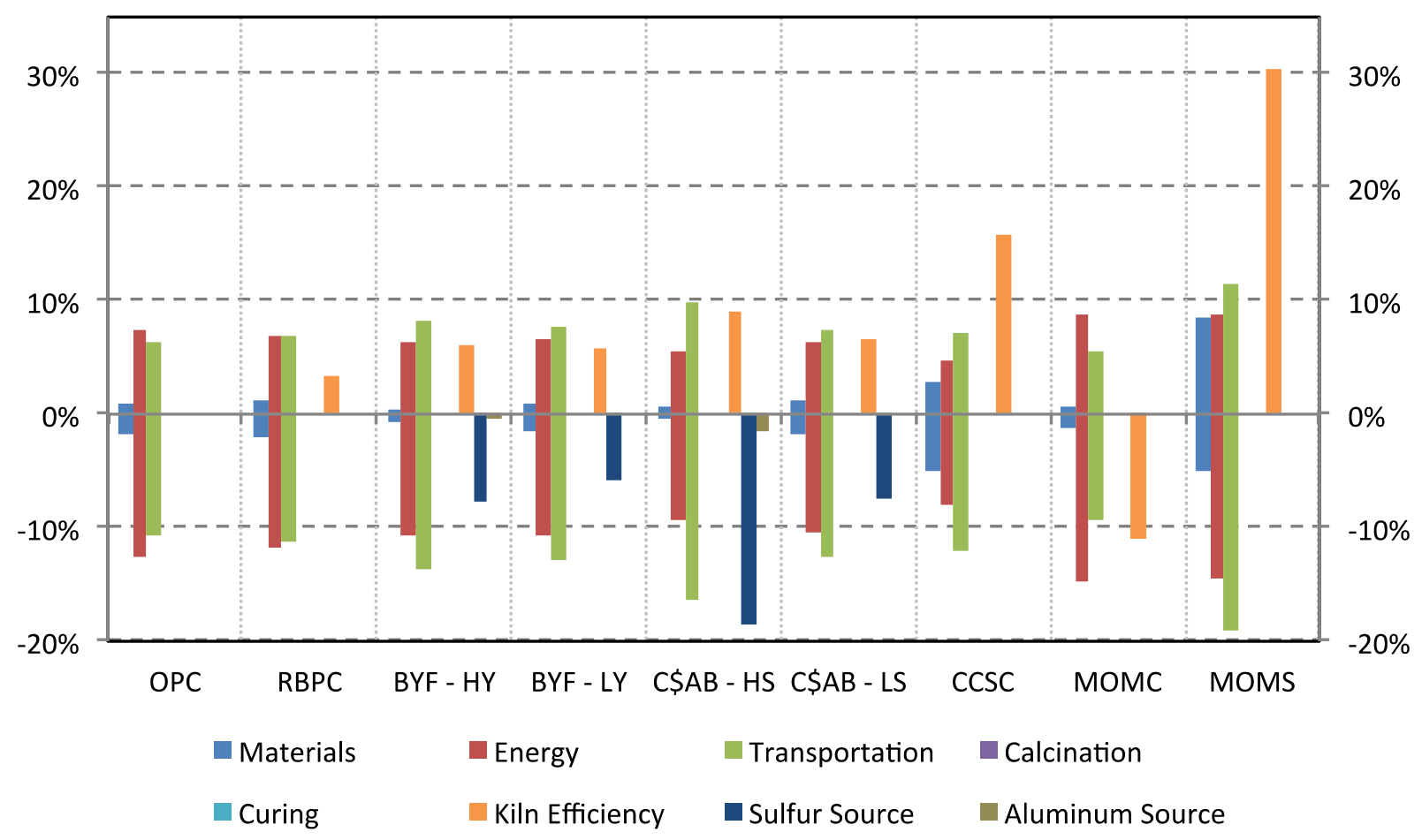

Figure S1. Sensitivity analysis of ozone depletion from ordinary portland cement and alternative cement binders

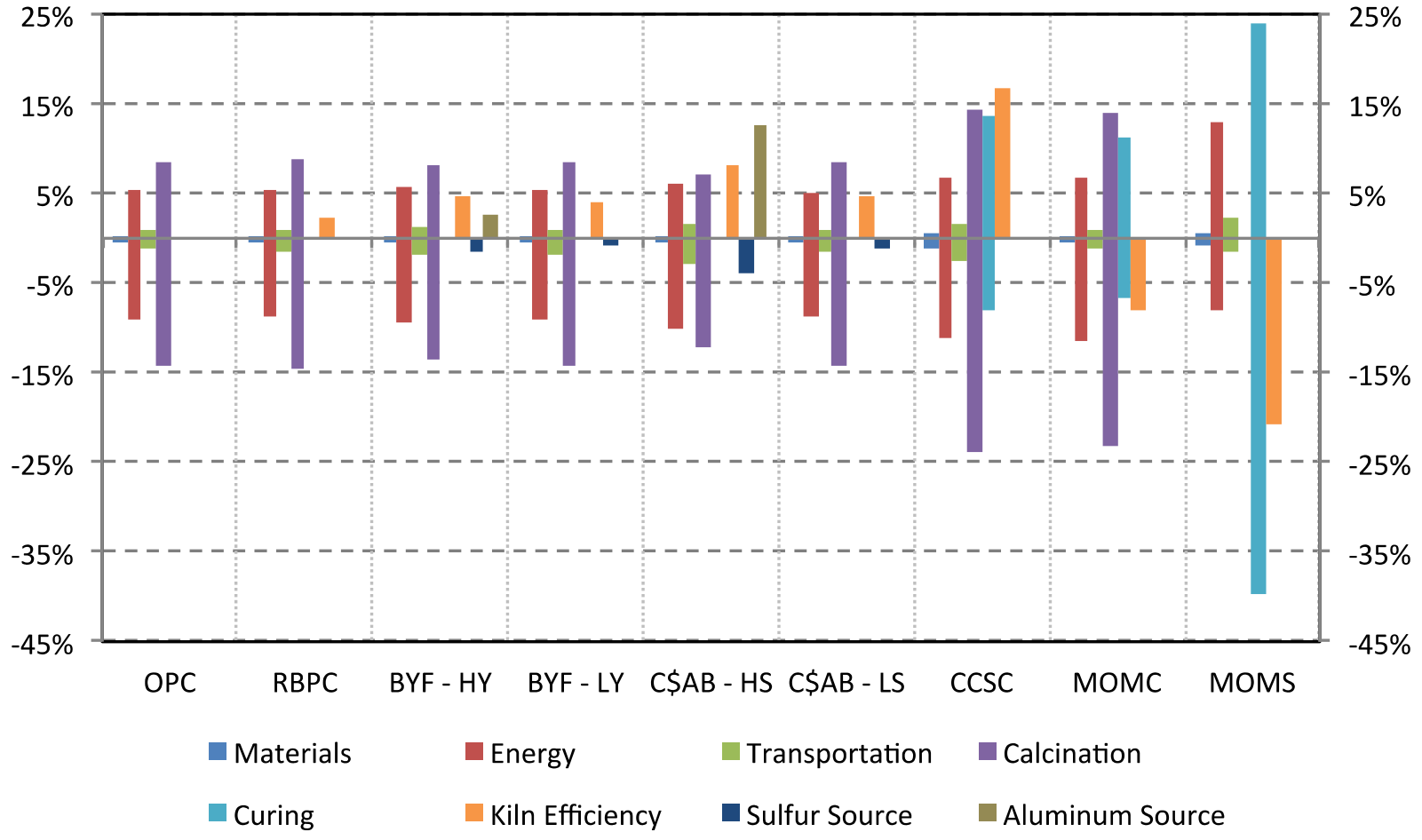

Figure S2. Sensitivity analysis of global warming potential from ordinary portland cement and alternative cement binders 


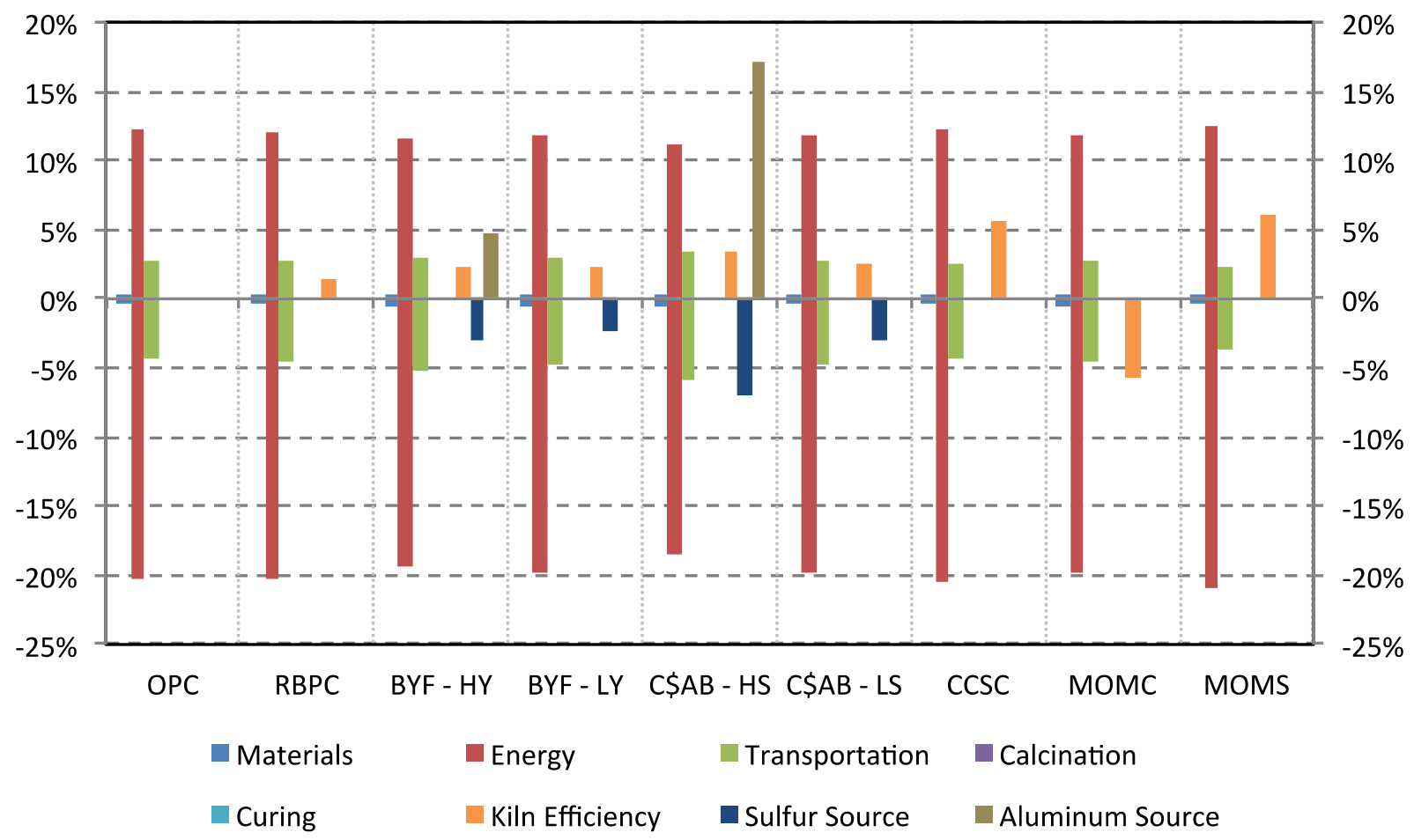

Figure S3. Sensitivity analysis of smog from ordinary portland cement and alternative cement binders

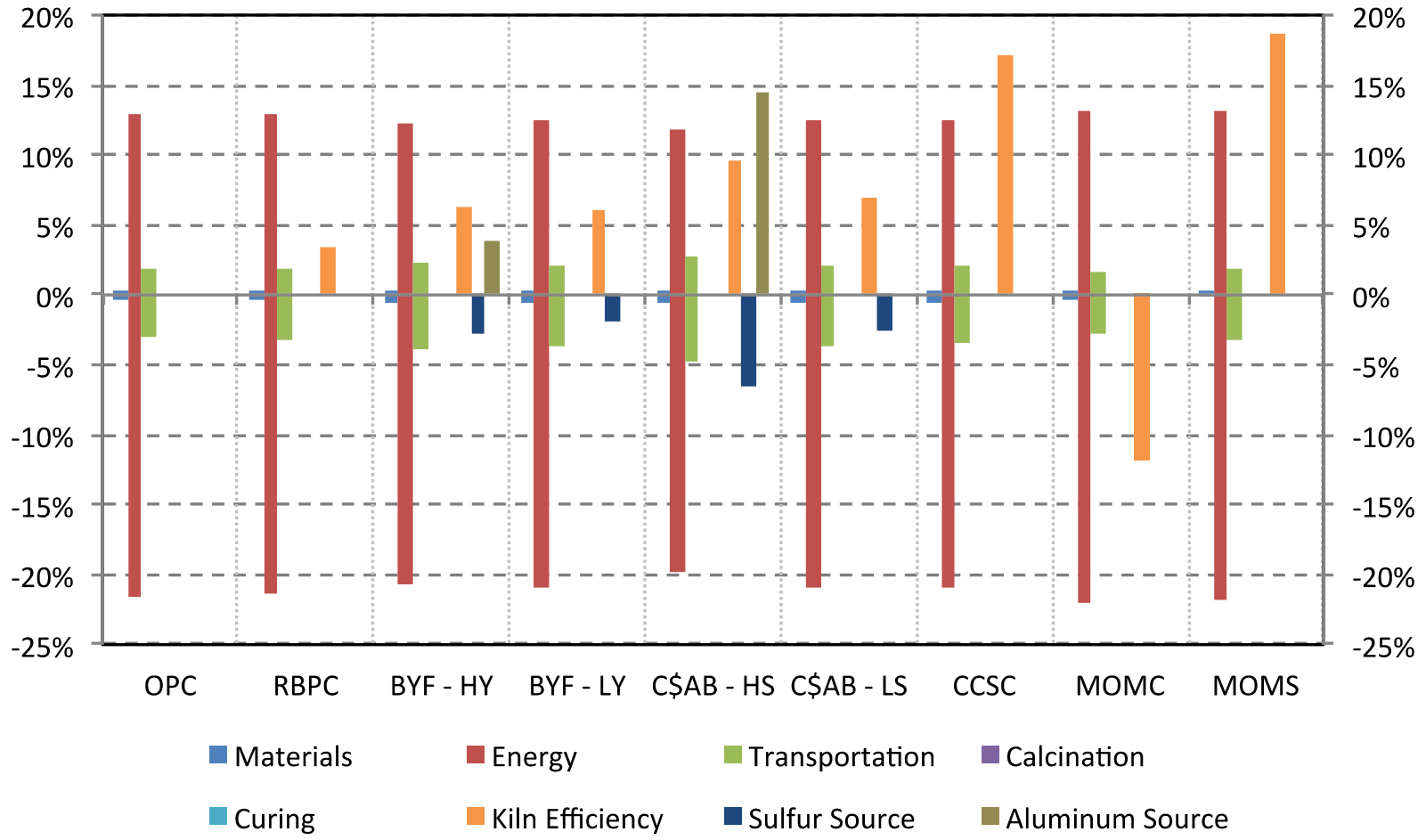

Figure S4. Sensitivity analysis of acidification from ordinary portland cement and alternative cement binders 


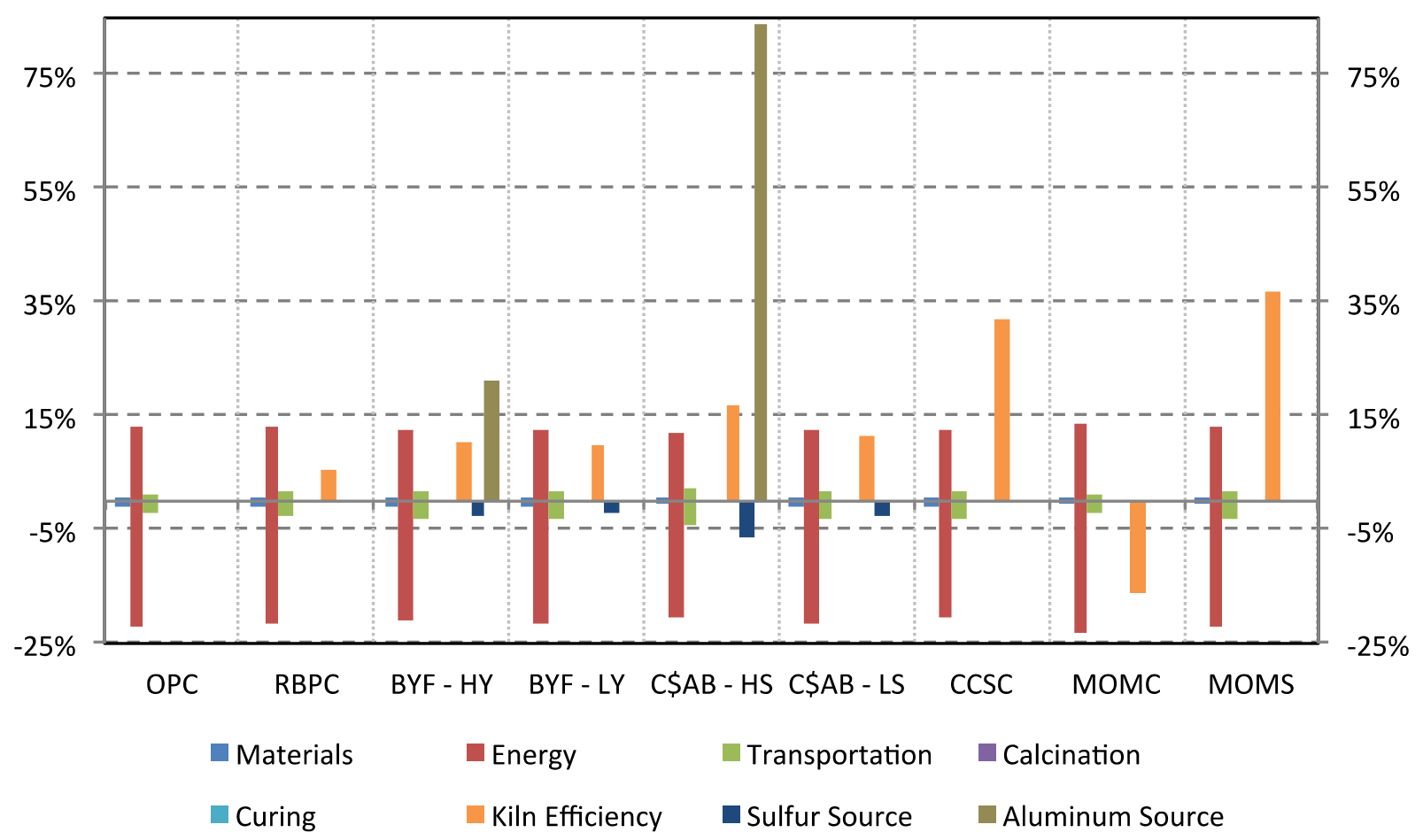

Figure S5. Sensitivity analysis of eutrophication from ordinary portland cement and alternative cement binders

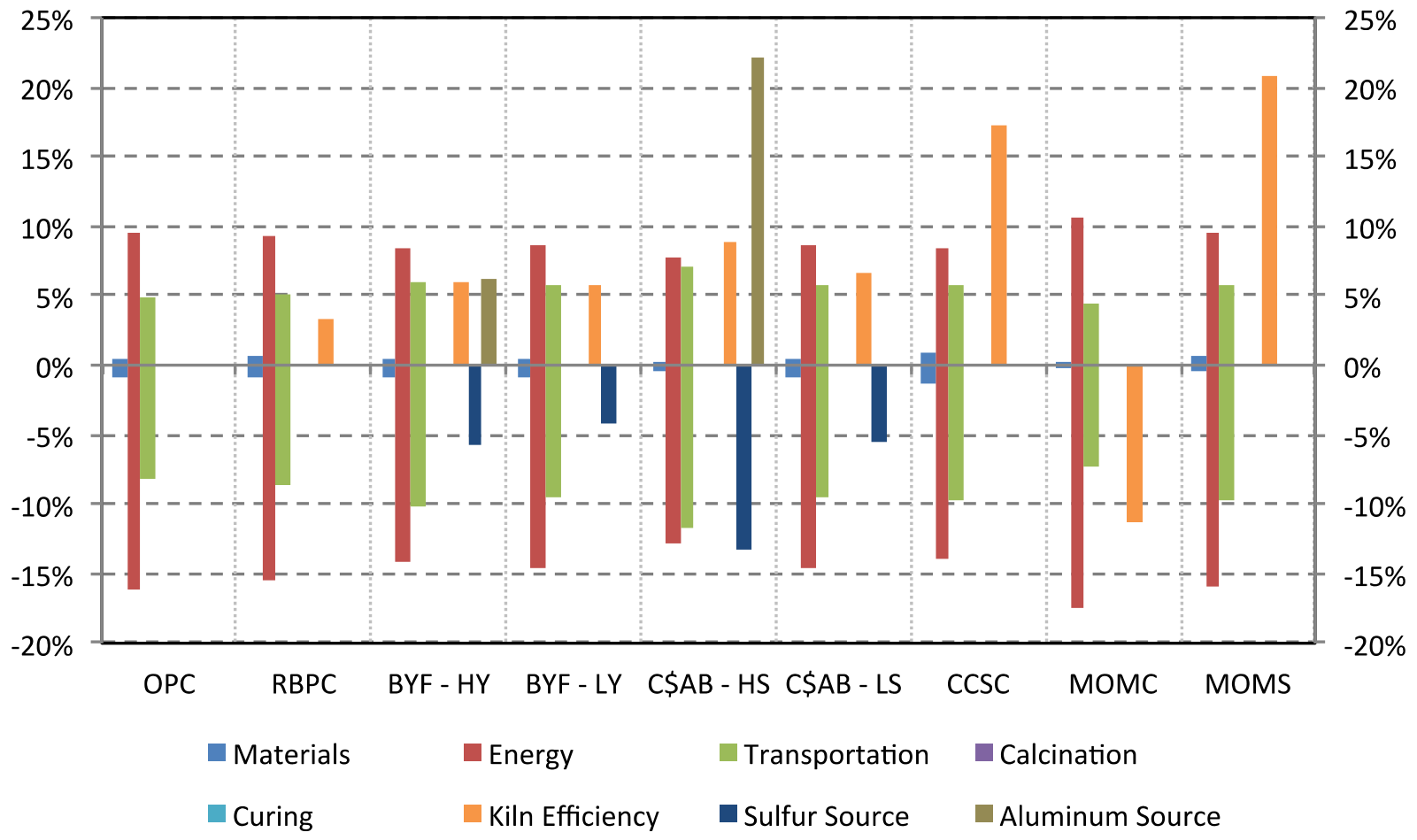

Figure S6. Sensitivity analysis of carcinogenics from ordinary portland cement and alternative cement binders 


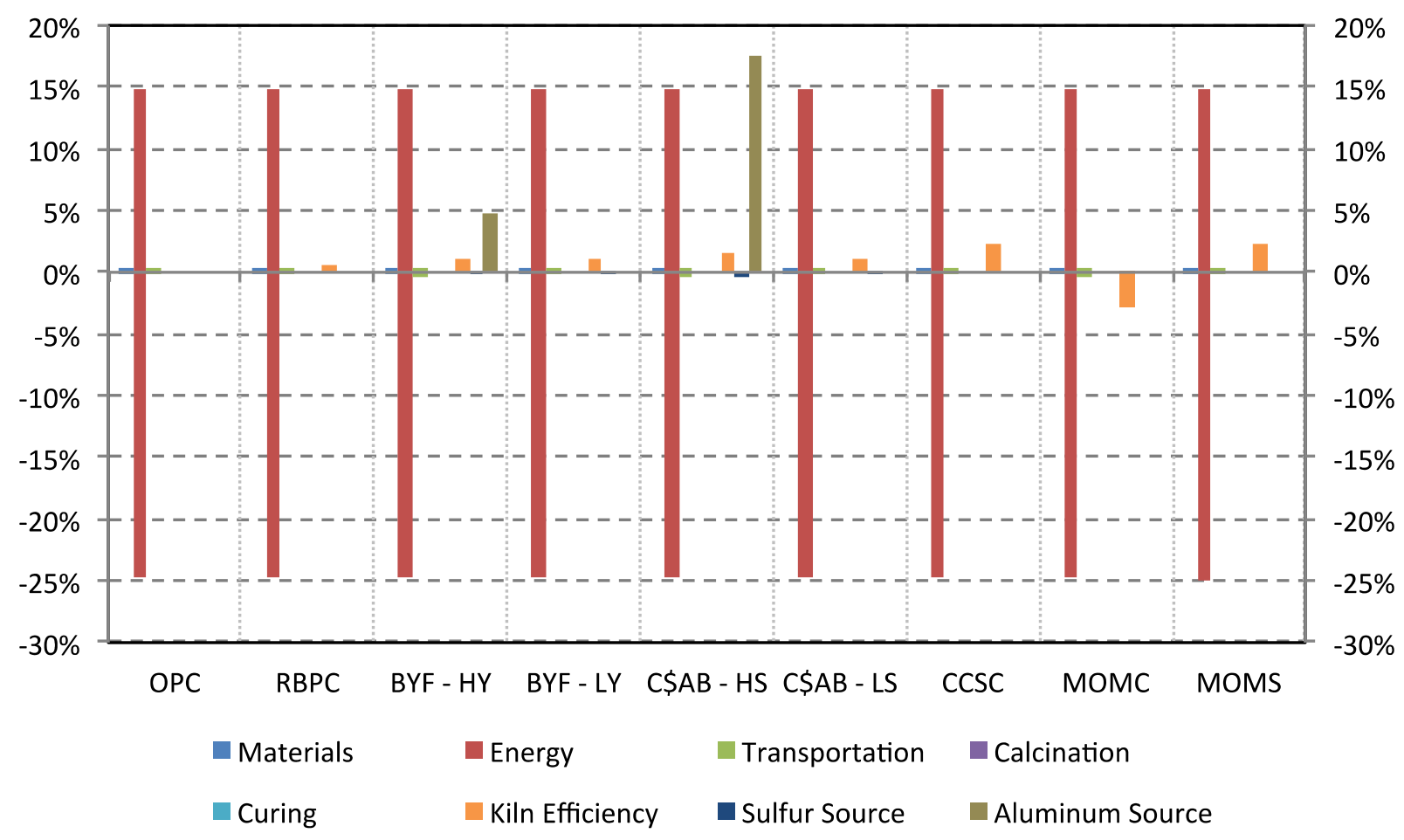

Figure S7. Sensitivity analysis of non-carcinogenics from ordinary portland cement and alternative cement binders

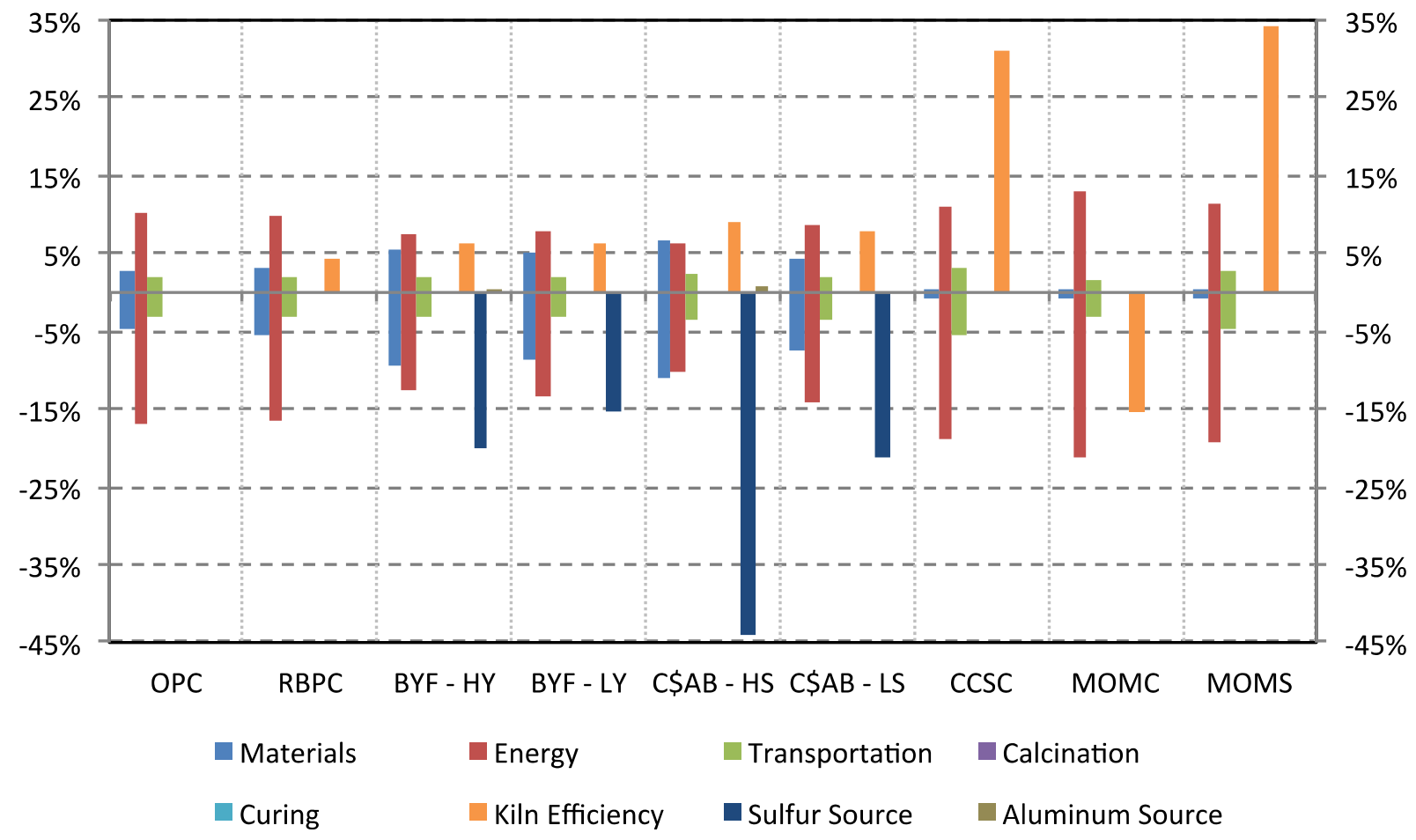

Figure S8. Sensitivity analysis of respiratory effects from ordinary portland cement and alternative cement binders 


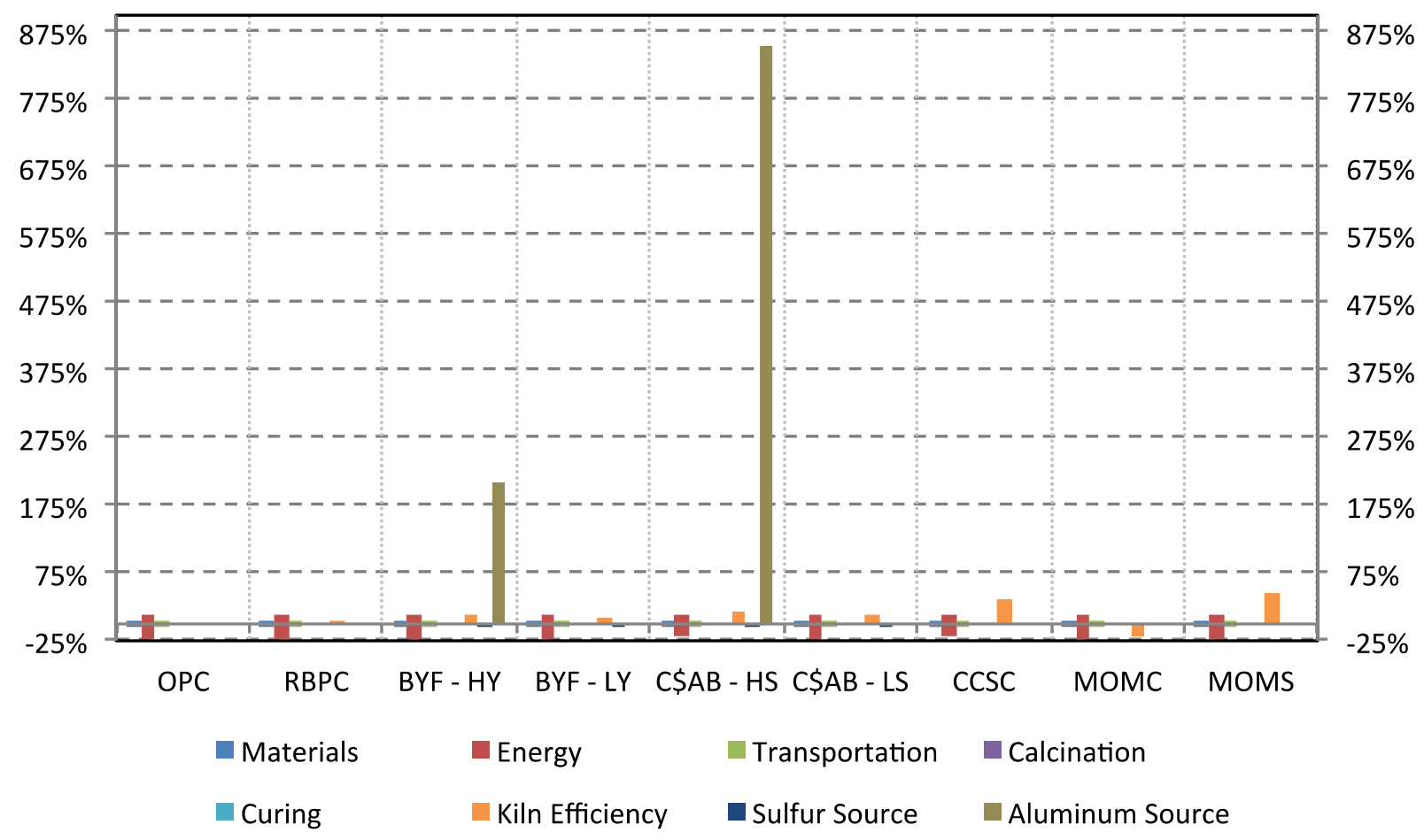

Figure S9. Sensitivity analysis of eco-toxicity from ordinary portland cement and alternative cement binders

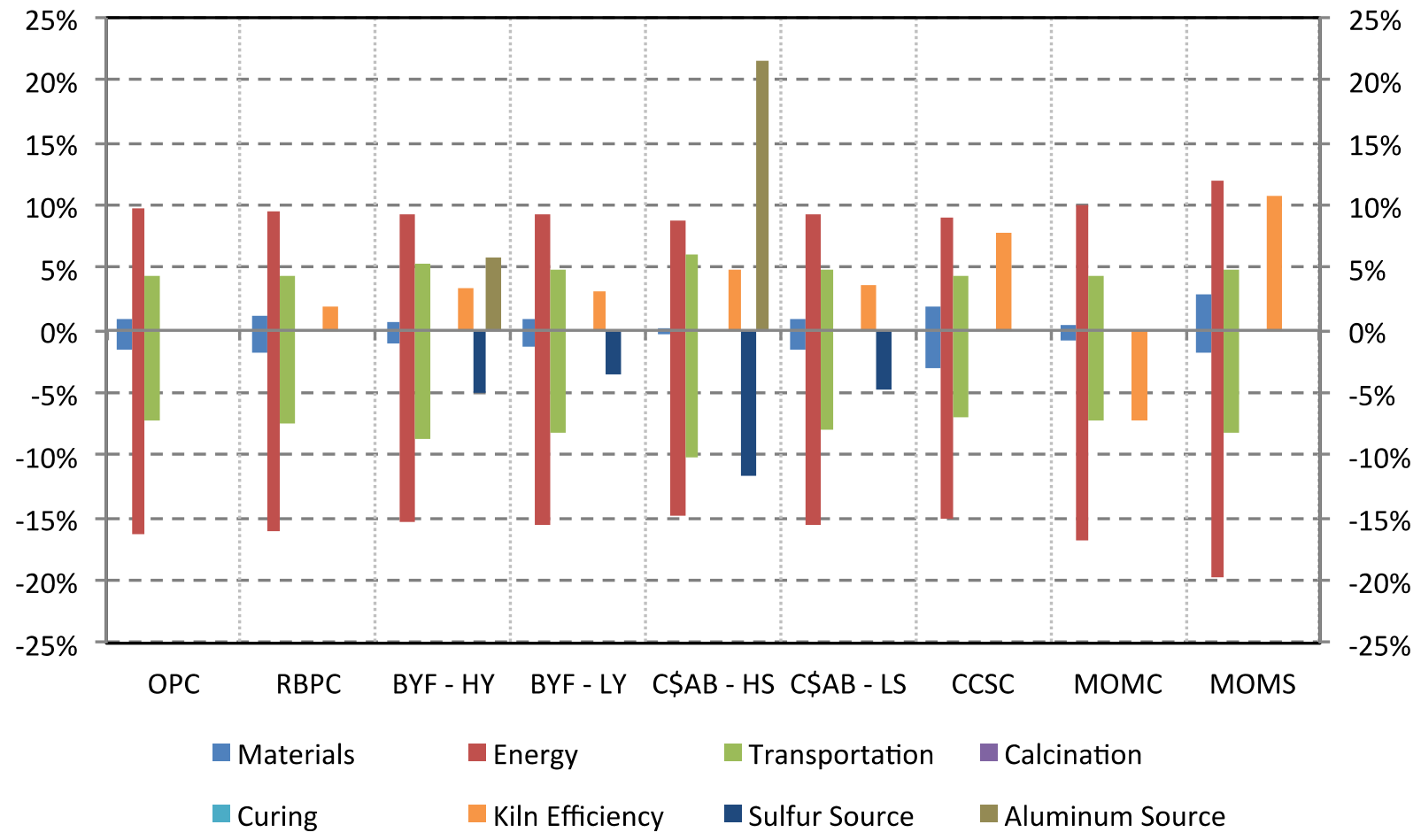

Figure S10. Sensitivity analysis of fossil fuel depletion from ordinary portland cement and alternative cement binders 